\title{
Che colpa abbiamo noi
}

\author{
di Andrea Sangiovanni and Nina Quarenghi
}

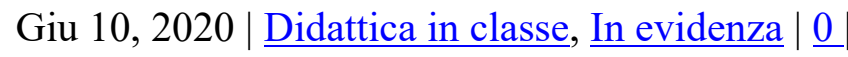

\section{La musica nella costruzione dell'identità collettiva giovanile negli anni Sessanta}

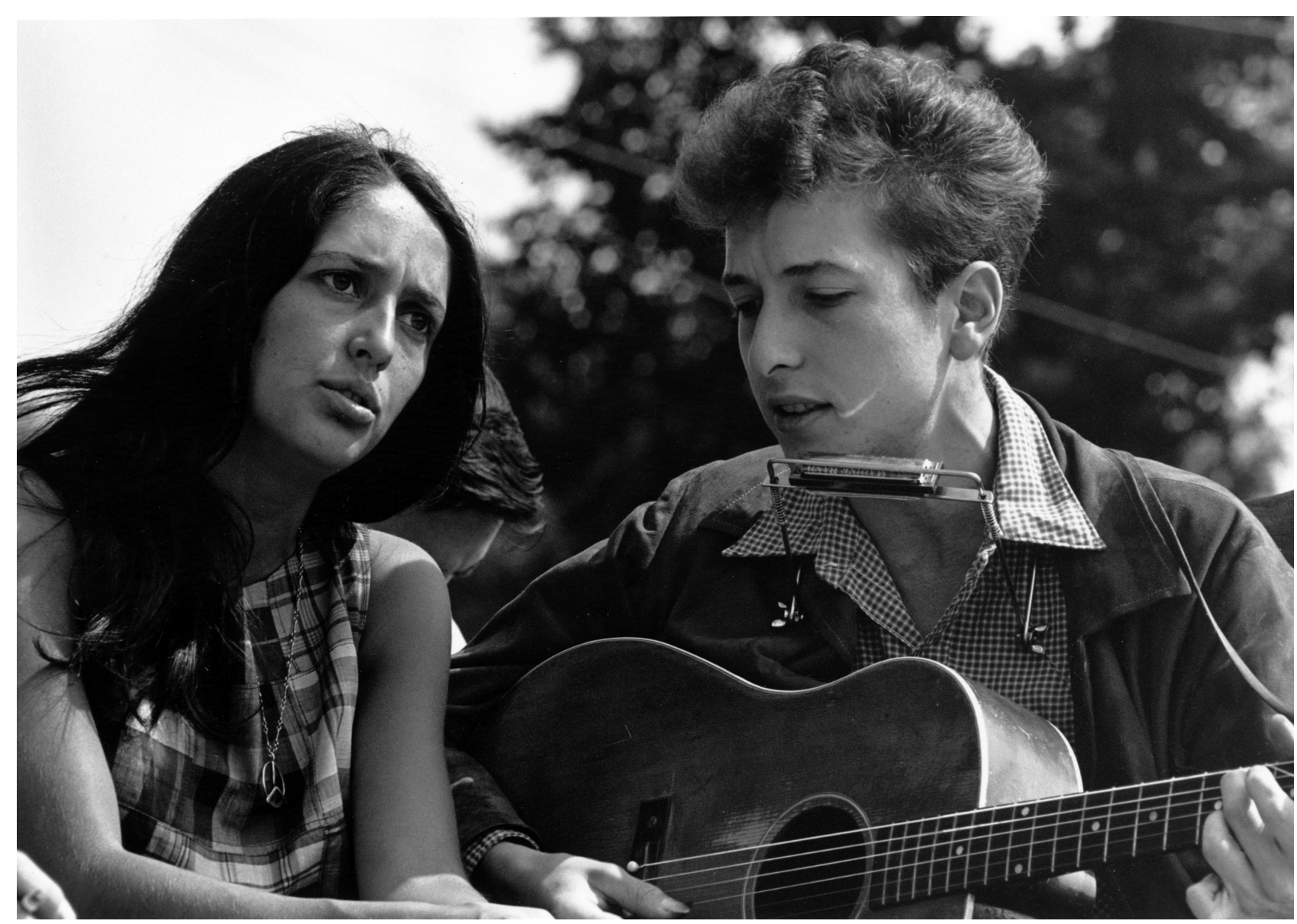

Joan Baez e Bob Dylan durante la Civil Rights March a Washington, D.C., il 28 agosto 1963. Foto di Rowland Scherman - U.S. National Archives and Records Administration, Pubblico dominio, Collegamento

\begin{abstract}
Questa attività didattica, rivolta alle classi di scuola secondaria di primo e secondo grado, ha come focus la cultura popolare, in particolare la musica. A partire dalla nascita di nuovi generi musicali, ragionando sugli strumenti di diffusione come i dischi, i jukebox, le band e la radio, e approdando ai megaconcerti di Woodstock e Altamont, si arriverà a delineare quel processo di costruzione di un'identità giovanile collettiva, avvenuto anche grazie al linguaggio comune della musica, che porterà alle grandi rivoluzioni del Sessantotto.

Nell'articolo sono presenti alcuni brani musicali, di cui si consiglia l'ascolto, mentre nella parte conclusiva viene proposta un'esercitazione di approfondimento, basata sull'ascolto e l'analisi di tre canzoni, che può essere svolta dagli studenti anche a distanza, utilizzando una scheda di analisi
\end{abstract}




\section{$\underline{\text { Scarica la scheda di analisi }}$}

\section{UN NUOVO MODO DI ESSERE GIOVANI}

Ogni rivoluzione si può paragonare all'eruzione di un vulcano, che sconvolge il paesaggio e lo lascia modificato per sempre al cessare della sua azione violenta, ma, proprio come lo scoppio improvviso è preceduto dal lento formarsi nel sottosuolo delle condizioni fisiche che portano all'esplosione, così le rivoluzioni sono l'epilogo di processi culturali latenti, lievitati nel tempo. Ciò si è verificato anche per il Sessantotto: l'insieme degli eventi che sconvolse il mondo in quell'anno è la conseguenza della crescita di sensibilità comuni nei giovani, avvenuta nei due decenni precedenti, e della loro volontà di partecipare alla vita sociale, economica e politica del mondo in trasformazione.

Dagli anni Cinquanta, negli Stati Uniti prima, in Europa e in Italia qualche anno più tardi, emerge un nuovo soggetto sociale, autonomo e produttore di cultura: i giovani. Non che i ragazzi di età compresa tra la pubertà e la maturità non fossero esistiti prima, ma mentre fino alla seconda guerra mondiale si tratta solo di un dato anagrafico, ora la generazione del baby-boom, cioè dei nati tra la metà degli anni Quaranta e i primi anni Cinquanta, periodo in cui, con l'aumento dei matrimoni, si registra una sensibile crescita demografica, comincia ad avere delle caratteristiche che la distinguono nettamente da chi li ha preceduti: questi giovani, grazie alla fase di sviluppo accelerato del mondo occidentale, godono di una maggiore disponibilità economica, possono proseguire gli studi e fruire di nuovi prodotti culturali, molti dei quali creati apposta per loro. Il termine che negli Stati Uniti li identifica è teenager, coloro che, nati dopo la guerra, frequentano le scuole superiori e le università tra gli anni Cinquanta e Sessanta.

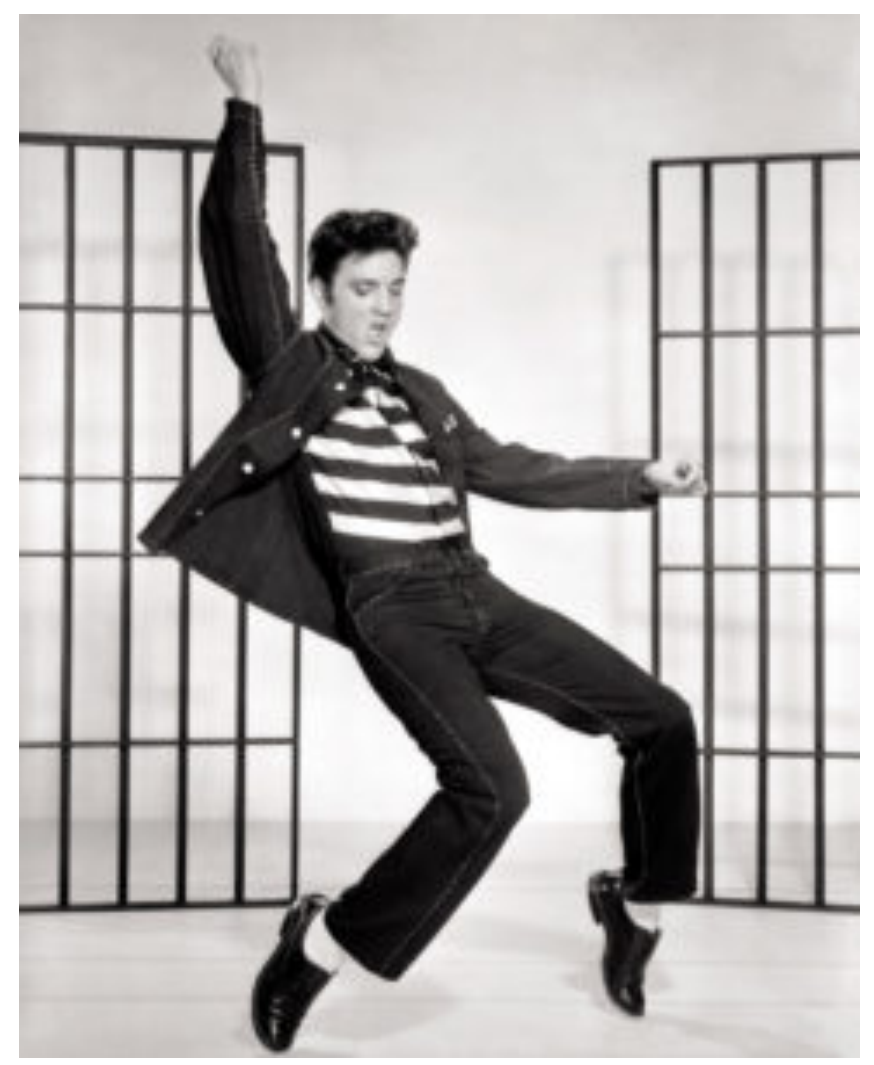

Elvis Presley in una fotografia promozionale del film "Jailhouse Rock". Di Metro-GoldwynMayer, Inc. - http://www.doctormacro.com/Images/Presley,\%20Elvis/Annex/Annex\%20\%20Presley,\%20Elvis\%20(Jailhouse\%20Rock) 01.jpg, Pubblico dominio, Collegamento 


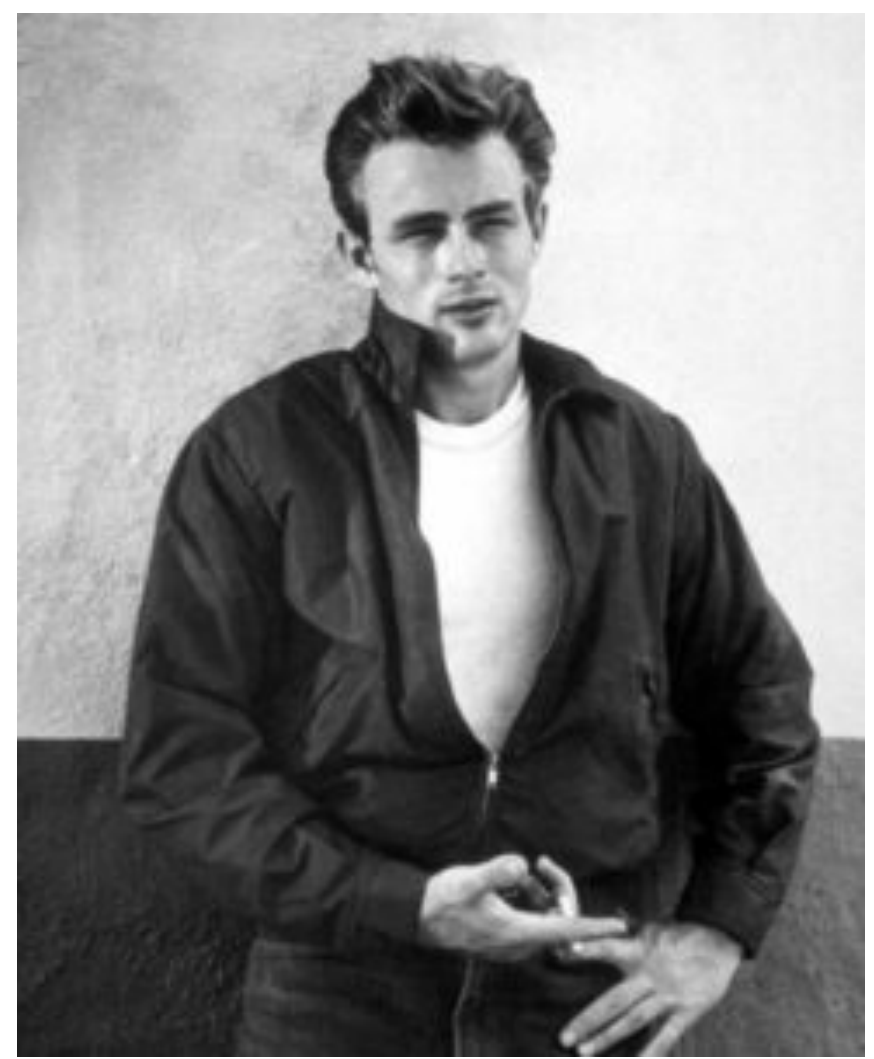

James Dean in una foto per il film "Gioventù bruciata" (Rebel Without a Cause) - Di Inhouse publicity still - Warner Bros. publicity still for for the film Rebel Without a Cause, Public Domain, Link

La storia della musica e quella del cinema si legano strettamente alla nascita di questo nuovo soggetto collettivo, perché è in questi ambiti culturali che si creano i modelli con i quali i giovani cominciano a identificarsi, primo fra tutti Elvis Presley. È il 1956 quando il giovane cantante esordisce in un programma televisivo visto in tutti gli Stati Uniti, l'Ed Sullivan Show; si può dire che con questa esibizione nasca "ufficialmente" il rock and roll, nuovo genere musicale associato alla figura di Elvis Presley e al suo modo di muoversi sul palco. Con il ritmo sfrenato delle sue canzoni e i movimenti suadenti del bacino - per quei tempi al limite dello scandalo - Elvis "the Pelvis" rompe tutti gli schemi e dimostra ai teenagers che, oltre ad esserci un nuovo modo di fare musica, esiste la possibilità di rivendicare la propria giovinezza anche attraverso un uso del corpo anticonformista e provocatorio.

Il cinema, a sua volta, racconta di giovani come il protagonista di Rebel without a cause (Nicholas Ray, Usa, 1955; titolo in italiano Gioventù bruciata) rappresentato dall'attore James Dean, "the bad boy from a good family", che incarna la quintessenza dell'adolescente temuto dagli adulti e dell'idolo venerato dai giovani. Un altro film di quegli anni, Blackboard jungle (Richard Brooks, Usa, 1955, titolo italiano Il seme della violenza) racconta di un professore democratico che si trova a insegnare a studenti aggressivi in una scuola molto difficile e ha la particolarità di avere come colonna sonora proprio Rock around the clock, la canzone simbolo del rock and roll e, per analogia, dei giovani disobbedienti e indomiti.

Gli adulti mostrano un sentimento ambivalente nei confronti di questa generazione: la sfruttano come nuovo segmento di mercato, attraverso la moda e le produzioni cinematografiche, ma allo stesso tempo la temono e tentano di controllarla. Anche per i giovani, tuttavia, esiste un rapporto 
ambiguo nei confronti delle novità culturali proposte da cinema e musica: da una parte le alimentano, dall'altra, pur cibandosene, le subiscono; da qui il rapporto conflittuale dei giovani di queste generazioni con i nuovi beni culturali e di consumo nei quali sono immersi.

\section{LA MUSICA È GIOVANE}

Tra gli anni Cinquanta e Sessanta la musica contagia tutti i giovani, grazie ai nuovi mezzi di diffusione, che contribuiscono a cambiare il modo di stare insieme. Nasce e di diffonde in quegli anni un disco in vinile di piccolo formato, il 45 giri, che contiene una canzone per lato, adatto per essere ascoltato, a basso costo, nei jukebox, apparecchi installati in bar o luoghi pubblici, che permettono di scegliere e attivare con una moneta un brano musicale. Capita che intorno ai jukebox i ragazzi si radunino non solo per ascoltare, ma anche per ballare e questa semplice azione, connaturata all'essere giovane, comporta delle novità: non si tratta più di balli di coppia, con ruoli ben stabiliti tra maschi e femmine, ma individuali; ciò significa che si balla insieme ma senza toccarsi e senza che il ragazzo guidi la ragazza, così ognuno trova un modo di esprimere la propria personalità individuale.

Con i jukebox avviene anche un'altra trasformazione. In un'Italia ancora profondamente divisa in classi, succede che intorno a queste "macchine urlanti" - così chiamate in modo dispregiativo dai critici adulti - si aggreghino ragazzi di tutte le categorie sociali: studenti e operai, artigiani e impiegati. Ciò che li accomuna è il riconoscersi nella stessa musica, che li rappresenta, perché è essa stessa giovane.

\section{LA MUSICA È INDUSTRIA}

Comincia a diffondersi, a partire dalla fine degli anni Cinquanta anche nelle famiglie italiane, il giradischi; come per il jukebox non si tratta solo di uno strumento, ma di un oggetto simbolico intorno al quale si crea una socialità che prima non esisteva: chi possiede un giradischi lo mette a disposizione degli altri e lo utilizza con una serie di cautele, che creano una ritualità nell'azione dell'ascoltare: si estrae il disco dalla sua custodia e siccome è un oggetto fragile, va usato con cura: viene spolverato perché non faccia "saltare" la puntina che lo deve leggere, lo si pone sul piatto e lo si avvia, per ascoltarlo poi interamente, tutti insieme, leggendo nel contempo i testi presenti all'interno della copertina. Tutto questo crea un'abitudine alla musica, un ascolto intensivo e condiviso, capace di fare comunità.

Un nuovo modo collettivo di stare insieme è dato anche dalle band: a metà degli anni Sessanta complessi e orchestrine sono più di seimila in Italia e continuano ad aumentare. Mentre prima di allora chi suonava uno strumento musicale doveva fare un percorso di studio esclusivo, ora basta che acquisti una chitarra e impari a suonarla in modo amatoriale unendosi ad altri giovani. Nascono anche i concorsi nazionali per band emergenti, spesso sponsorizzati proprio dalle riviste musicali espressamente dedicate ai teenagers; in Italia hanno questi nomi: Big, Ciao amici, Ciao 2001,

Giovani.

Dagli elementi fin qui esposti si può notare come la musica dei giovani del baby-boom sia motore di trasformazioni culturali e contemporaneamente industriali, perché crea continuamente una domanda di beni di consumo, dai dischi ai giradischi, dagli strumenti musicali alle riviste specializzate, prima inesistenti. 


\section{L'IMPORTANZA DELLA RADIO}

Fin dalla sua nascita la radio è stato uno strumento privilegiato per l'ascolto della musica e anche negli anni Sessanta acquista un ruolo fondamentale nella diffusione della musica per i giovani, grazie ad alcune trasmissioni dedicate. I nuovi generi musicali sono osteggiati dai vertici della Rai, che considerano questi ritmi troppo sfrenati, quindi pericolosi, diseducativi e dannosi per le giovani generazioni; essendo la Rai l'unica emittente del Paese, è necessario che trasmetta valori, idee e principi che nella musica rock non si trovano. D'altro canto ci sono delle necessità industriali: i giovani rappresentano un nuovo target e vanno assecondati e conquistati per trarne profitto. In seguito a queste spinte nascono delle trasmissioni che, in qualche modo, confortano le ansie degli adulti, accontentano i giovani e fanno crescere l'economia. Una di queste è Bandiera gialla.

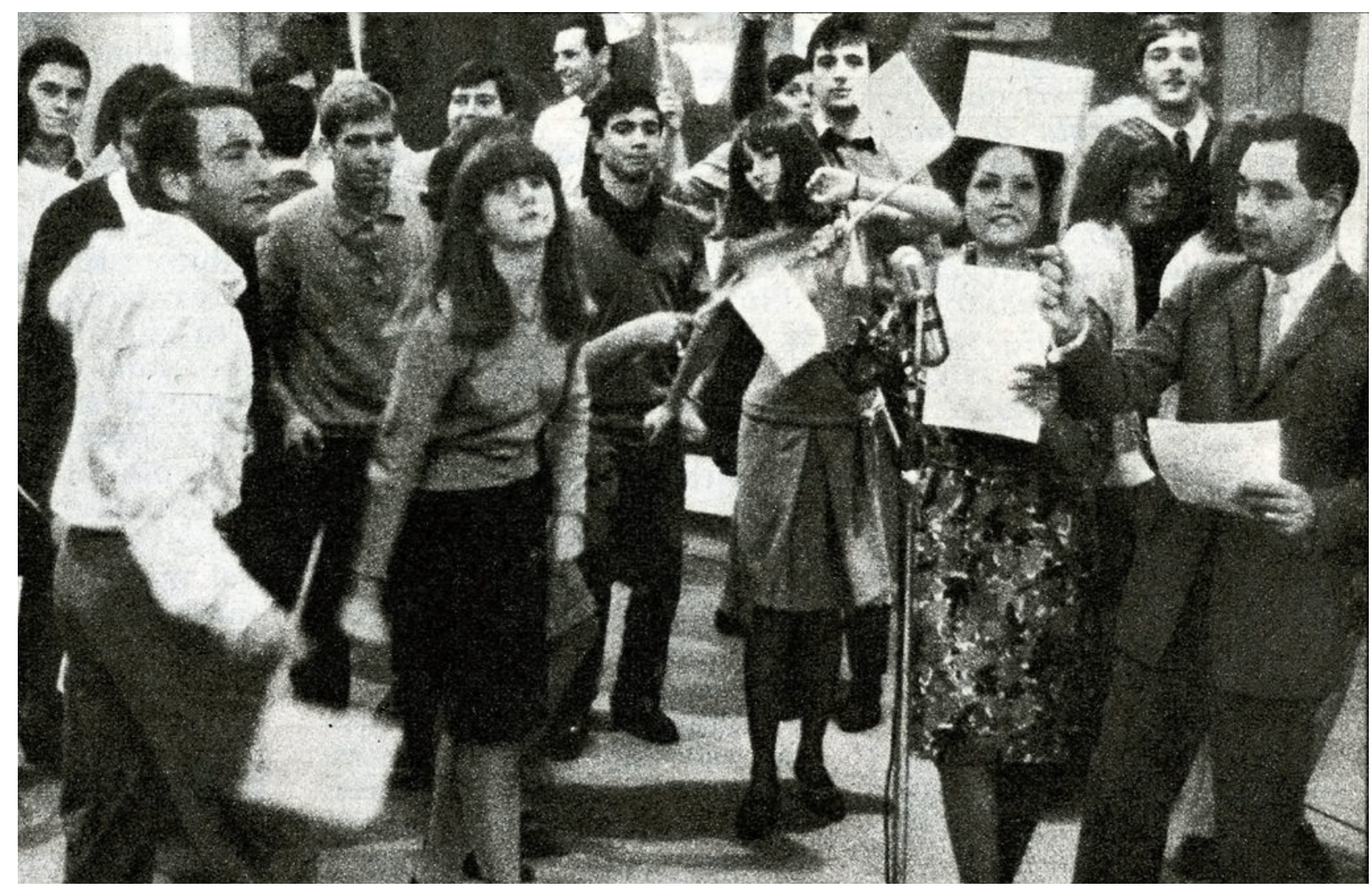

Bandiera gialla - Di Unknown author - Radiocorriere, Public Domain, Link

Bandiera gialla nasce nel 1966 con Gianni Boncompagni e Renzo Arbore, due giovani conduttori radiofonici con una lunga carriera di successo davanti a loro, prima nella radio - che rivoluzionano con le loro innovazioni - poi in televisione. Durante le trasmissioni venivano chiamati in studio gruppi di ragazzi per ascoltare e votare canzoni nuove, italiane o straniere; il brano vincitore veniva trasmesso anche la settimana successiva. Il nome della trasmissione, che, come diceva Boncompagni all'inizio di ogni puntata, «era severamente vietata ai maggiori di diciotto anni», è molto significativo: la bandiera gialla è, in nautica, il segnale che indica una pestilenza a bordo, un luogo potenzialmente pericoloso, una zona circoscritta da cui è impossibile uscire. Con questo escamotage i conduttori riescono a fare accettare ai vertici della Rai la messa in onda di canzoni, che altrimenti sarebbe stato difficile ascoltare: esse venivano trasmesse solo durante Bandiera gialla, programma in cui i giovani, come animali in gabbia, erano liberi di muoversi ma non di scappare. 
Negli stessi anni nasce Per voi giovani, trasmissione di ascolto delle novità musicali, che diventa, dall'inizio degli anni Settanta, con la conduzione di Paolo Giaccio e Mario Luzzatto Fegiz, una vera e propria tribuna per i ragazzi: essi avevano l'occasione di fare sentire la loro voce nel momento in cui i conduttori li intervistavano o li facevano confrontare su temi a loro cari, come le occupazioni delle scuole o delle fabbriche e le motivazione della loro protesta. Questa trasmissione avrà una sorta di trasposizione in televisione; nasce in particolare Speciale per voi $T V$, luogo in cui i personaggi più in auge, come per esempio Lucio Battisti, cantano in diretta e si fanno intervistare dai ragazzi del pubblico; il tono delle domande di questi giovani, talvolta, è così diretto da far sembrare le interviste quasi degli interrogatori.

\section{BEATLES NELLA COSTRUZIONE DI UNA IDENTITÀ COLLETTIVA}

Uno degli elementi fondamentali per la creazione di un'identità giovanile collettiva è rappresentato dalla musica dei Beatles. Il celebre quartetto inglese non è importante solo per le trasformazioni musicali, che genera nel breve arco di dieci anni, ma anche perché incarna la musica che tutti ascoltano.

I Beatles vengono in Italia nel 1965 per una breve tournée, facendo tappa a Milano, Genova e Roma; al termine di uno di questi concerti alcune ragazze rilasciano una testimonianza: «Noi siamo state al concerto dei Beatles e per la prima volta abbiamo capito cosa significa essere giovani». Sono ragazze, ma solo in quel momento acquistano la consapevolezza di essere giovani in quanto parte di qualcosa che prima di allora era indefinito e astratto: il loro essere giovani si concretizza in quelle voci, in quelle note, in quelle quattro figure sul palco, nelle loro movenze, abbigliamento e pettinatura, e in quella folla di ragazzi che, come loro, amano questa musica e chi l'ha creata. I Beatles riescono dunque a delineare e ad ampliare una identità collettiva, altrimenti confusa $\mathrm{e}$ inespressa.

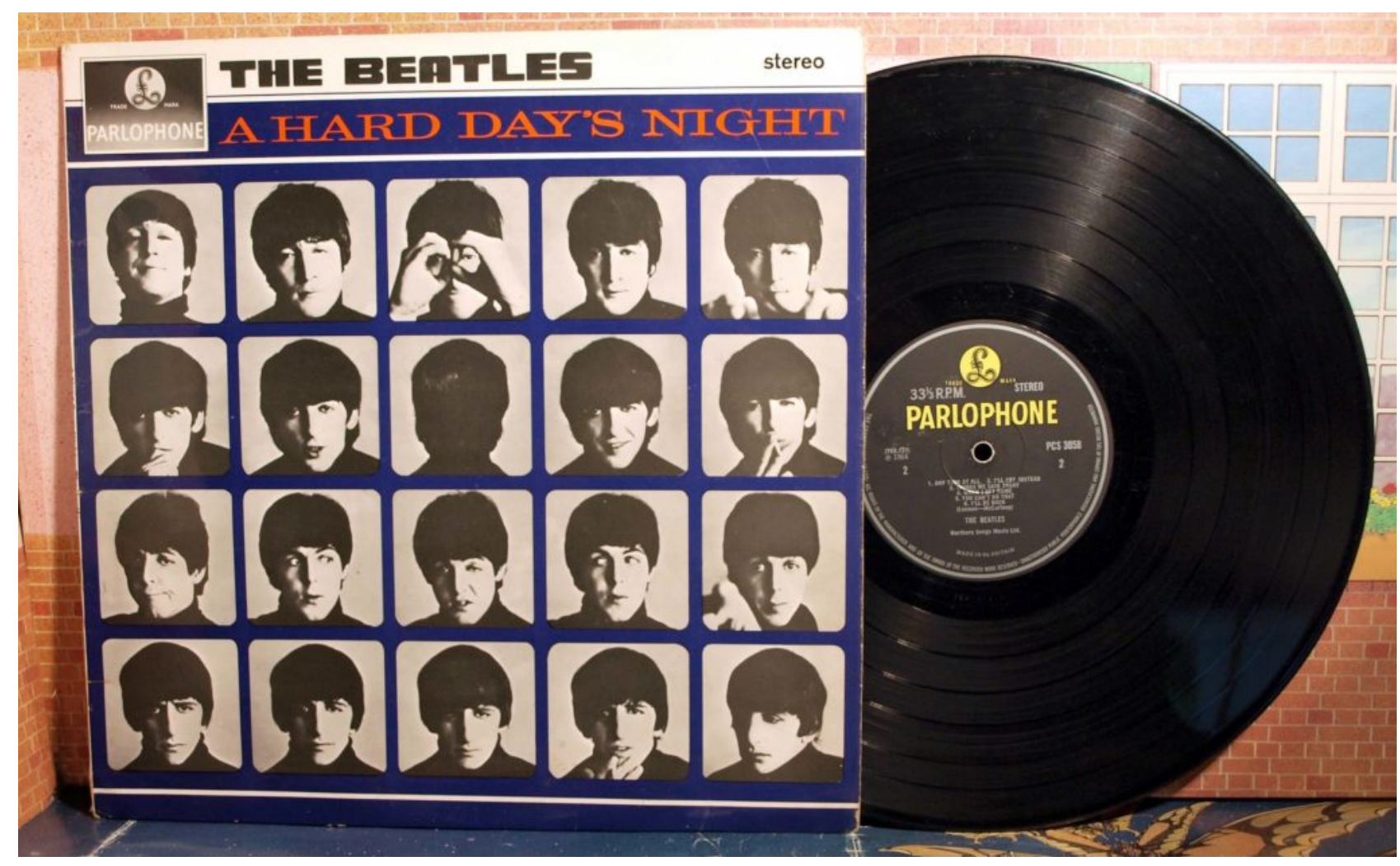


Sono talmente compenetrati al mondo giovanile cui si rivolgono, che le loro canzoni subiscono delle trasformazioni sorprendenti per bocca dei loro fan, come accade in occasione delle prime manifestazioni studentesche a Berkley, in California. Nell'autunno-inverno del 1964 gli studenti occupano l'Università; la loro lotta politica è sintetizzata nel nome del movimento nato in quella stagione, chiamato Free Speech Movement, uno degli antenati, se così si può dire, delle agitazioni degli anni successivi; la "presa di parola" sarà infatti una delle caratteristiche del Sessantotto. Quando, a Berkley, accade che il rettore fa sgomberare i banchetti politici all'interno del Campus, si crea una manifestazione studentesca e migliaia di ragazzi marciano verso l'aula magna; in questa folla si sentono canzoni di lotta, una delle quali viene intonata sulla melodia di un famosissimo brano dei Beatles: A Hard day's night. Nelle voci degli studenti le parole vengono cambiate in: «It'll be a long hard fight». Accade qui una cosa molto speciale: questi ragazzi non cantano inni tradizionali di protesta, né adattano le parole del Movimento sulle classiche arie folk utilizzate in queste occasioni, ma si servono di una canzone contemporanea, peraltro d'amore, e la trasformano in senso politico: «Sarà una lunga e dura battaglia».

I Beatles, in quel momento, sono lontani dalla lotta politica (anche se qualche anno più tardi alcuni dei componenti della band vi prenderanno parte, in particolare John Lennon), tuttavia il loro modo di rispecchiare l'essere giovani diventa una chiave di lettura della realtà per questi ragazzi che, in occasione delle proteste, esprimono loro stessi e la loro identità, il loro credo politico, ricalcando la musica dei Beatles in cui si identificano.

\section{UNA MUSICA NUOVA}

[Si propone l'ascolto di Blowin' in the wind (1966)]

Quante strade deve percorrere un uomo prima che lo si possa chiamare uomo?

Sì, e quanti mari deve sorvolare una bianca colomba prima che possa riposare sulla sabbia?

Sì, e quante volte le palle di cannone dovranno volare prima che siano bandite per sempre?

Sì, e quanti anni può esistere una montagna prima di essere spazzata fino al mare?

Sì, e quanti anni la gente deve vivere prima che possa essere finalmente libera?

Sì, e quante volte un uomo può voltare la testa fingendo di non vedere?

Sì, e quante volte un uomo deve guardare verso l'alto prima che riesca a vedere il cielo?

Sì, e quante orecchie deve avere un uomo prima che possa ascoltare la gente piangere?

Sì, e quante morti ci vorranno perché egli sappia che troppe persone sono morte?

Negli Stati Uniti nasce in questi anni una musica impegnata, i cui interpreti più famosi sono Bob Dylan e Joan Baez. Blowin' in the wind, è forse la canzone più celebre del cantautore, che si ispira a una lunga tradizione di musica folk politica, ma utilizzata in senso nuovo. Bob Dylan scrive questa canzone nel momento in cui viene acclamato come colui che può, attraverso le sue canzoni, fornire una risposta alle inquietudini del suo tempo. La grandezza di questo brano, che lo rende un classico perché trasversale a ogni epoca, è proprio nella sua incapacità di dare risposte, di trovare soluzioni alle storture dell'uomo, all'ingiustizia mascherata da buone intenzioni. L'agitazione nell'animo di Dylan è la stessa dei ragazzi del Free Speech Movement, degli studenti che manifestano contro la guerra del Vietnam, o contro l'autoritarismo nelle scuole, perché dietro a queste motivazioni più o 
meno contingenti ci sono domande esistenziali profonde, relative al rapporto tra le forze che regolano il mondo e, più in generale, alla prepotenza dell'uomo verso il suo simile.

Questa musica politica è diversa dalle precedenti, perché ha un respiro più ampio e un portato poetico diverso. Non a caso Bob Dylan è stato insignito del Premio Nobel per la Letteratura nel 2016.

\section{IN ITALIA}

[Si propone l'ascolto di Che colpa abbiamo noi (1966)]

La canzone scelta è particolare perché cantata dai Rokes, una band inglese, che esegue cover di canzoni americane in italiano. Negli anni Sessanta è prassi diffusa, da parte dei complessi italiani, tradurre le canzoni provenienti da Stati Uniti e Inghilterra per portare queste melodie e questi ritmi nuovi in Italia. I Rokes sono un gruppo beat; il termine deriva dalla beat generation, quel movimento giovanile che si sviluppò in campo artistico e letterario negli anni Cinquanta negli Stati Uniti; in Italia il portato di questa corrente culturale viene recepito qualche anno più tardi e in modo diverso: il beat è soprattutto un modo di fare musica, che si innesta su un genere in voga nei primissimi anni Sessanta, la cosiddetta musica yé-yé, ma ha caratteristiche del tutto nuove. Come dice Caterina Caselli, una delle cantanti simbolo di questa stagione, «lo yé-yé è un genere allegro, spensierato, carino, mentre il beat è un genere che batte in quattro il tempo, che si fa con la voce arrabbiata, è tutta un'altra cosa». Cambia il modo di fare musica, dal momento che si sta modificando lo spirito giovanile collettivo, e ciò avviene progressivamente, dapprima in modo latente, quasi impercettibile, poi sempre più marcato.

Una canzone emblematica di questi anni prerivoluzionari è proprio quella di cui si è proposto l'ascolto, Che colpa abbiamo noi: la melodia è leggera e scanzonata, ma se si presta attenzione al testo troviamo qualcosa di nuovo: c'è il malcontento dei giovani per la società ingiusta ereditata dalla generazione che li ha preceduti e che, oltretutto, li incolpa dei mali che lei stessa ha creato; c'è la consapevolezza di subire un mondo sbagliato e la volontà di volerlo migliorare. I temi del Sessantotto stanno prendendo corpo, lievitano nelle canzoni, nel modo in cui vengono diffuse all'interno del mondo giovanile, mentre il divario con gli adulti, autoritari e chiusi nella loro rigidità, cresce sempre più.

\section{SANREMO 1967: UN TRAUMA CULTURALE}

Nel 1967, durante il Festival di Sanremo, evento musicale come sempre atteso da tutta la società italiana, accade un fatto tragico, che rappresenta un vero e proprio trauma culturale per un'intera generazione: il cantante Luigi Tenco, escluso dalla finale, si suicida. Lascia un biglietto con questo messaggio: «Io ho voluto bene al pubblico italiano e gli ho dedicato inutilmente cinque anni della mia vita. Faccio questo non perché sono stanco della vita (tutt'altro) ma come atto di protesta contro un pubblico che manda Io tu e le rose in finale e ad una commissione che seleziona La rivoluzione. Spero che serva a chiarire le idee a qualcuno. Ciao. Luigi».

[img] 
La canzone proposta da Tenco, Ciao amore, ciao, è apparentemente una canzone d'amore, ma in realtà racconta le trasformazioni sociali e culturali del Paese: affronta il tema delle grandi migrazioni interne, dalle aree povere della campagna meridionale al triangolo industriale; parla del senso di insicurezza dei giovani che abbandonano la propria vita, sacrificando gli affetti, per partecipare alla costruzione di una modernità da cui, irrimediabilmente, restano esclusi. Questo testo impegnato, mascherato da canzone popolare, non viene apprezzato dalla giuria del Festival, che evidentemente ha un altro metro di misura: sceglie, tra le canzoni scartate, La rivoluzione, che, a dispetto del titolo, è tutt'altro che rivoluzionaria. Ecco infatti le parole della prima strofa:

Guarda quante facce scure

Piene di rancore

Sono ferme là

Guarda quei ragazzi uniti

Tutti colorati

Stan correndo qua

Ma che succederà?

$\mathrm{Ci}$ sarà la rivoluzione

Nemmeno un cannone però tuonerà

$\mathrm{Ci}$ sarà la rivoluzione

L'amore alla fine vedrai vincerà

E basteranno pochi anni oppure poche ore

Per fare un mondo migliore

Un mondo dove tutti saranno perdonati

Chi ha vinto e chi ha perduto

Vedrai si abbraccerà.

(La rivoluzione, voce di Gianni Pettenati, testo di Mogol)

È contro questa ipocrisia che Tenco sacrifica la propria vita. Il giorno dopo la sua morte, il Festival prosegue come se nulla fosse e al funerale, a parte Fabrizio De André e pochi altri, il mondo dello spettacolo è assente. Tutto questo rimarca la validità del suo messaggio: il malessere espresso nei versi della sua canzone rappresenta il lato B del progresso e dell'ubriacatura dei nuovi beni di consumo; il pubblico non vuole sentirne parlare e d'altra parte la giuria sa che il Festival della canzone italiana è specchio del Paese e bisogna esaltare l'immagine di un'Italia serena, moderna, innamorata; quell'anno vincono Claudio Villa e Iva Zanicchi, con una classica canzone d'amore.

Il gesto di Luigi Tenco, tuttavia, lascia un segno nella cultura musicale italiana: da quel momento si rafforza e diventa dominante un modo di fare musica, che prima esisteva ma in modo solo marginale, quello delle canzoni d'autore. I cantautori saranno i veri protagonisti del panorama musicale italiano negli anni Settanta e l'azione estrema di Tenco sarà narrata proprio da due dei maggiori rappresentanti: Fabrizio De André, che gli dedica Preghiera in gennaio (1967) e, un decennio più tardi, Francesco De Gregori, che scrive per lui Festival (1976).

\section{MEGACONCERTI: LA COSTRUZIONE DI UN'IDENTITÀ GIOVANILE}

Il lento processo culturale, attraverso il quale, a partire dagli anni Cinquanta, i giovani emergono come nuovo soggetto sociale e politico, porta allo scoppio di una rivoluzione, alla fine degli anni Sessanta, che, in qualche modo, coinvolge tutto il mondo. Il Sessantotto è un evento internazionale, senza però essere unitario: ogni realtà locale si muove autonomamente, a seconda degli interessi e 
delle situazioni contingenti, ma le "parole d'ordine" sono senz'altro comuni: l'essere giovani e lottare contro l'autoritarismo e la guerra, volere fare sentire la propria voce e combattere per un mondo più equo. La musica, ancora una volta, rappresenta il linguaggio comune per i giovani rivoluzionari e veicola il loro modo di sentire, anche attraverso eventi musicali come i megaconcerti, che si tengono negli Stati Uniti e in Inghilterra, e che funzionano come riti di costruzione dell'identità collettiva. Già dalla metà degli anni Sessanta ci sono grandi raduni costruiti intorno alla musica, basti pensare alle "Summer of love" di San Francisco nel 1967, il cui emblema è la canzone di Scott McKenzie If you are going to San Francisco.

La sola idea di andare a questi eventi, di partecipare vestiti - o svestiti - allo stesso modo, l'uso delle droghe come ricerca di un nuovo modo esprimere sé stessi, e, soprattutto, la musica rock di artisti ormai leggendari: tutto questo fa da collante per un mondo giovanile, che gli adulti continuano a non capire e a disprezzare.

[Si propone l'ascolto dell'assolo alla chitarra di Jimi Hendrix a Woodstock - 1969]

Woodstock, agosto 1969. Jimi Hendrix, distorcendo con la sua chitarra elettrica l'inno nazionale degli Stati Uniti, realizza una doppia operazione dal grande significato simbolico, che il milione di giovani spettatori capisce al volo: rende omaggio agli Stati Uniti, glorifica il grande Paese di cui tutti loro fanno parte, che rende possibile la realizzazione di questo festival sterminato, ma allo stesso tempo svela le storture del Paese, campione dell'imperialismo che schiaccia popolazioni più deboli, come i civili in Vietnam.

\section{ATTIVITà DIDATTICA}

Per meglio comprendere i nuclei tematici contenuti nell'articolo, proponiamo agli studenti una esercitazione, che può essere svolta anche a distanza, che consiste nell'ascolto di tre canzoni, nella compilazione di una scheda di analisi e nella restituzione e condivisione delle considerazioni fatte durante lo svolgimento del compito. Sono stati scelti questi tre brani musicali perché, pur essendo diversi per ritmo, melodia e voci, sono rappresentativi degli anni che precedono la rivoluzione del Sessantotto.

\section{Caterina Caselli, Nessuno mi può giudicare (1966)}

La verità mi fa male, lo so

La verità mi fa male, lo sai

Nessuno mi può giudicare, nemmeno tu

(La verità ti fa male, lo so)

Lo so che ho sbagliato una volta e non sbaglio più

(La verità ti fa male, lo so)

Dovresti pensare a me

E stare più attento a te

C'è già tanta gente che ce l'ha su con me, chi lo sa perché?

Ognuno ha il diritto di vivere come può

(La verità ti fa male, lo so)

Per questo una cosa mi piace e quell'altra no

(La verità ti fa male, lo so)

Se sono tornata a te

Ti basta sapere che 
Ho visto la differenza tra lui e te

Ed ho scelto te

Se ho sbagliato un giorno ora capisco che

L'ho pagata cara la verità

Io ti chiedo scusa, e sai perché?

Sta di casa qui la felicità

Molto, molto più di prima io t'amerò

In confronto all'altro sei meglio tu

E d'ora in avanti prometto che

Quel che ho fatto un dì non farò mai più

Ognuno ha il diritto di vivere come può

(La verità ti fa male, lo so)

Per questo una cosa mi piace e quell'altra no

(La verità ti fa male, lo so)

Se sono tornata a te

Ti basta sapere che

Ho visto la differenza fra lui e te

Ed ho scelto te

Se ho sbagliato un giorno ora capisco che

L'ho pagata cara la verità

Io ti chiedo scusa, e sai perché?

Sta di casa qui la felicità

Nessuno mi può giudicare, nemmeno tu

\section{Scott McKenzie, If you are going to San Francisco (1967)}

Se stai andando a San Francisco

Assicurati di mettere dei fiori tra i capelli

Se stai andando a San Francisco

Lì incontrerai persone gentili

Per quelli che vengono a San Francisco

L'estate sarà un love-in

Nelle strade di San Francisco

Persone gentili con fiori tra i capelli

Attraverso tutta la nazione

Come una strana vibrazione

C'è un'intera generazione

Con una nuova spiegazione

Gente in movimento, gente in movimento

Per quelli che vengono a San Francisco

Assicuratevi di mettere dei fiori tra i capelli

Se vieni a San Francisco

L'estate sarà un love-in

\section{I Nomadi, Dio è morto (1967)}

Ho visto

La gente della mia età andare via 
Lungo le strade che non portano mai a niente

Cercare il sogno che conduce alla pazzia

Nella ricerca di qualcosa che non trovano

Nel mondo che hanno già, dentro alle notti che dal vino son bagnate

Lungo le strade da pastiglie trasformate

Dentro le nuvole di fumo del mondo fatto di città

Essere contro ad ingoiare la nostra stanca civiltà

E un Dio che è morto

Ai bordi delle strade, Dio è morto

Nelle auto prese a rate, Dio è morto

Nei miti dell'estate, Dio è morto

Mi han detto

Che questa mia generazione ormai non crede

In ciò che spesso han mascherato con la fede

Nei miti eterni della patria o dell'eroe

Perché è venuto ormai il momento di negare

Tutto ciò che è falsità, le fedi fatte di abitudine e paura

Una politica che è solo far carriera

Il perbenismo interessato la dignità fatta di vuoto

L'ipocrisia che di chi sta sempre con la ragione e mai col torto

E un Dio che è morto

Nei campi di sterminio, Dio è morto

Coi miti della razza, Dio è morto

Con gli odi di partito, Dio è morto

Ma penso

Che questa mia generazione è preparata

A un mondo nuovo e a una speranza appena nata

Ad un futuro che ha già in mano

A una rivolta senza armi

Perché noi tutti ormai sappiamo

Che se Dio muore è per tre giorni e poi risorge

In ciò che noi crediamo, Dio è risorto

In ciò che noi vogliamo, Dio è risorto

Nel mondo che faremo, Dio è risorto

\section{Bibliografia}

- A. Banti, Wonderland, Laterza, Roma-Bari, 2017

- P. Cavallo, P. Iaccio (a cura di), Penso che un sogno così non ritorni mai più. L'Italia del miracolo tra storia, cinema, musica e televisione, Liguori, Napoli, 2016

- F. Fasce, La musica nel tempo. Una storia dei Beatles, Einaudi, Torino, 2018

- D. Giachetti, Anni Sessanta, comincia la danza, BFS, Pisa, 2002

- P. Mattera, Questioni di metodo: gli audiovisivi come agenti e come fonti? Un caso di studio: la musica popolare degli anni Sessanta, in "Officina della Storia", 2/2017 
(https://www.officinadellastoria.eu/it/2017/10/17/questioni-di-metodo-gli-audiovisivi-comeagenti-e-come-fonti-un-caso-di-studio-la-musica-popolare-negli-anni-sessanta/) 